\title{
Saturation physics at HERA and RHIC: An unified description
}

\author{
V.P. Gonçalves ${ }^{a, *}$, M.S. Kugeratski ${ }^{b}$, M.V.T. Machado ${ }^{c}$, F.S. Navarra ${ }^{b}$ \\ ${ }^{a}$ High and Medium Energy Group (GAME), Instituto de Física e Matemática, Universidade Federal de Pelotas Caixa Postal 354, \\ CEP 96010-900, Pelotas, RS, Brazil \\ ${ }^{\mathrm{b}}$ Instituto de Física, Universidade de São Paulo, C.P. 66318, 05315-970 São Paulo, SP, Brazil \\ ${ }^{c}$ High Energy Physics Phenomenology Group, GFPAE, IF-UFRGS Caixa Postal 15051, CEP 91501-970 Porto Alegre, RS, Brazil
}

Received 4 August 2006; received in revised form 17 October 2006; accepted 26 October 2006

Editor: J.-P. Blaizot

\begin{abstract}
One of the frontiers of QCD which are intensely investigated in high energy experiments is the high energy (small $x$ ) regime, where we expect to observe the non-linear behavior of the theory. In this regime, the growth of the parton distribution should saturate, forming a color glass condensate (CGC). In fact, signals of parton saturation have already been observed both in $e p$ deep inelastic scattering at HERA and in deuteron-gold collisions at RHIC. Currently, a global description of the existing experimental data is possible considering different phenomenological saturation models for the two processes within the CGC formalism. In this Letter we analyze the universality of these dipole cross section parameterizations and verify that they are not able to describe the HERA and RHIC data simultaneously. We analyze possible improvements in the parameterizations and propose a new parameterization for the forward dipole amplitude which allows us to describe quite well the small- $x$ ep HERA data on $F_{2}$ structure function as well as the $d A u$ RHIC data on charged hadron spectra. It is an important signature of the universality of the saturation physics.

(c) 2006 Elsevier B.V. All rights reserved.
\end{abstract}

In the past few years much theoretical effort has been devoted towards the understanding of the high energy limit of the strong interaction theory (for recent reviews see, e.g. [1-3]). In the high energy limit, perturbative quantum chromodynamics (pQCD) predicts that the small- $x$ gluons in a hadron wavefunction should form a color glass condensate (CGC), which is described by an infinite hierarchy of the coupled evolution equations for the correlators of Wilson lines [4-7]. In the absence of correlations, the first equation in the Balitsky-JIMWLK hierarchy decouples and is then equivalent to the equation derived independently by Kovchegov within the dipole formalism [8]. The color class condensate is characterized by the limitation on the maximum phase-space parton density that can be reached in the hadron wavefunction (parton saturation), with the transition being specified by a typical scale, which is energy dependent

\footnotetext{
* Corresponding author.

E-mail address: barros@ufpel.edu.br (V.P. Gonçalves).
}

and is called saturation scale $Q_{\mathrm{s}}\left[Q_{\mathrm{s}}^{2} \propto A^{\alpha} x^{-\lambda}\right]$. Moreover, in the CGC formalism the dipole-target forward scattering amplitude $\mathcal{N}$ for a given impact parameter $\boldsymbol{b}$, which is directly related with the two-point function of Wilson lines, encodes all the information about the hadronic scattering, and thus about the non-linear and quantum effects in the hadron wave function. The function $\mathcal{N}$ can be obtained by solving the BalitskyJIMWLK evolution equation in the rapidity $Y \equiv \ln (1 / x)$ [9]. Its main properties are: (a) for the interaction of a small dipole $\left(\boldsymbol{r} \ll 1 / Q_{\mathrm{s}}\right), \mathcal{N}(\boldsymbol{r}) \approx \boldsymbol{r}^{2}$, implying that this system is weakly interacting; (b) for a large dipole $\left(\boldsymbol{r} \gg 1 / Q_{\mathrm{s}}\right)$, the system is strongly absorbed and therefore $\mathcal{N}(\boldsymbol{r}) \approx 1$. This property is associated to the large density of saturated gluons in the hadron wave function. Another remarkable feature of CGC formalism is that the dense, saturated system of partons to be formed in hadronic wave functions at high energy has universal properties, the same for all hadrons or nuclei.

In the CGC formalism the description of the observables is directly related to the behavior of $\mathcal{N}$. For instance, the $F_{2}$ 
structure function is probed in $e p(A)$ process and is given by $F_{2}^{p(A)}\left(x, Q^{2}\right)=\left(Q^{2} / 4 \pi^{2} \alpha_{\mathrm{em}}\right)\left(\sigma_{T}^{\gamma^{*} p(A)}+\sigma_{L}^{\gamma^{*} p(A)}\right)$, where [10]

$$
\begin{aligned}
\sigma_{L, T}^{\gamma^{*} p(A)}\left(x, Q^{2}\right)= & \sum_{f} \int d z d^{2} \boldsymbol{r}\left|\Psi_{L, T}^{(f)}\left(z, \boldsymbol{r}, Q^{2}\right)\right|^{2} \sigma_{\mathrm{dip}}(x, \boldsymbol{r}) \\
= & \sum_{f} \int d z d^{2} \boldsymbol{r}\left|\Psi_{L, T}^{(f)}\left(z, \boldsymbol{r}, Q^{2}\right)\right|^{2} \\
& \times 2 \int d^{2} \boldsymbol{b} \mathcal{N}_{F}(x, \boldsymbol{r}, \boldsymbol{b}),
\end{aligned}
$$

with $\mathcal{N}_{F}$ being the fundamental representation of the forward dipole amplitude, $\boldsymbol{r}$ defining the relative transverse separation of the pair (dipole) and $z(1-z)$ the longitudinal momentum fraction of the quark (antiquark). The photon wave functions $\Psi_{L, T}$ are determined from light cone perturbation theory (see e.g. Ref. [11]). It is useful to assume that the impact parameter dependence of $\mathcal{N}_{F}$ can be factorized as $\mathcal{N}_{F}(x, \boldsymbol{r}, \boldsymbol{b})=$ $\mathcal{N}_{F}(x, \boldsymbol{r}) S(\boldsymbol{b})$, so that $\sigma_{\mathrm{dip}}(x, \boldsymbol{r})=\sigma_{0} \mathcal{N}_{F}(x, \boldsymbol{r})$, with $\sigma_{0}$ being a free parameter related to the non-perturbative QCD physics. Similarly, the single-inclusive hadron production in hadronhadron processes is described in the CGC formalism by [12]

$$
\begin{aligned}
x_{F} \frac{d \sigma^{p p(A) \rightarrow h X}}{d x_{F} d^{2} p_{t} d^{2} \boldsymbol{b}} & \\
= & \frac{1}{(2 \pi)^{2}} \int_{x_{F}}^{1} d x_{p} \frac{x_{p}}{x_{F}}\left[f_{q / p}\left(x_{p}, Q_{f}^{2}\right) \mathcal{N}_{F}\left(\frac{x_{p}}{x_{F}} p_{t}, \boldsymbol{b}\right)\right. \\
& \quad \times D_{h / q}\left(\frac{x_{F}}{x_{p}}, Q_{f}^{2}\right)+f_{g / p}\left(x_{p}, Q_{f}^{2}\right) \mathcal{N}_{A}\left(\frac{x_{p}}{x_{F}} p_{t}, \boldsymbol{b}\right) \\
& \left.\quad \times D_{h / g}\left(\frac{x_{F}}{x_{p}}, Q_{f}^{2}\right)\right],
\end{aligned}
$$

where $p_{t}$ and $x_{F}$ are the transverse momentum and the Feynman- $x$ of the produced hadron, respectively. The variable $x_{p}$ denotes the momentum fraction of a projectile parton and $\boldsymbol{b}$ is the impact parameter. Moreover, $f\left(x_{p}, Q_{f}^{2}\right)$ is the projectile parton distribution functions and $D\left(z, Q_{f}^{2}\right)$ the parton fragmentation functions into hadrons. These quantities evolve according to the DGLAP [13] evolution equations and respect the momentum sum-rule. In Eq. (2), $\mathcal{N}_{F}(\boldsymbol{k}, \boldsymbol{b})$ and $\mathcal{N}_{A}(\boldsymbol{k}, \boldsymbol{b})$ are the fundamental and adjoint representations of the forward dipole amplitude in momentum space. The amplitudes $\mathcal{N}_{A, F}(\boldsymbol{k}, \boldsymbol{b})$ and $\mathcal{N}_{A, F}(\boldsymbol{r}, \boldsymbol{b})$ are directly related by a Fourier transform.

The search of signatures for the parton saturation effects has been an active subject of research in the last years. In particular, it has been observed that the HERA data at small $x$ and low $Q^{2}$ can be successfully described with the help of saturation models [14-19]. Moreover, experimental results for the total [20], diffractive [21] and inclusive charm cross sections [22,23] present the property of geometric scaling. On the other hand, the observed [24] suppression of high $p_{T}$ hadron yields at forward rapidities in $d A u$ collisions at RHIC had its behavior anticipated on the basis of CGC ideas [25]. A current shortcoming of these analyzes comes from the non-existence of an exact solution of the non-linear equation in the full kinematic range, which implies the construction of phenomenological models satisfying the asymptotic behavior which is under theoretical control. Several models for the forward dipole cross section have been used in the literature in order to fit the HERA and RHIC data. In particular, the phenomenological models from Refs. [14-17] have been proposed in order to describe the HERA data, while those from Refs. [12,26] have been able to describe the $d A u$ RHIC data. An important aspect should be emphasized at this point. Although at HERA it is possible to probe values of $x$ two orders of magnitude smaller than at RHIC, the saturation scales for these two scenarios are very similar due to the nuclear medium (see Fig. 1 in Ref. [27]). Consequently, one can expect to be possible to cross relate these experiments in this respect and gain a clear understanding of the CGC in high energy experiments. There are several similarities among the phenomenological models proposed in Refs. [12,14-17,26]. In particular, in these models the function $\mathcal{N}$ has been modeled in terms of a simple Glauber-like formula

$\mathcal{N}(x, \boldsymbol{r})=1-\exp \left[-\frac{1}{4}\left(\boldsymbol{r}^{2} Q_{s}^{2}(x)\right)^{\gamma\left(x, \boldsymbol{r}^{2}\right)}\right]$,

where $\gamma$ is the anomalous dimension of the target gluon distribution. The main difference comes from the predicted behavior for the anomalous dimension, which determines the transition from the non-linear to the extended geometric scaling regimes, as well as from the extended geometric scaling to the DGLAP regime. A detailed comparison has been presented in Ref. [27]. As the models from Refs. $[14,15,17]$ have been exhaustively discussed in the literature, in this Letter we only present a brief review of the models proposed in Refs. [12,26]. In the KKT model [26] the expression for the quark dipole-target forwardscattering amplitude is given by [26]:

$\mathcal{N}_{F}(\boldsymbol{r}, x)=1-\exp \left[-\frac{1}{4}\left(\boldsymbol{r}^{2} \bar{Q}_{s}^{2}\right)^{\gamma\left(Y, \boldsymbol{r}^{2}\right)}\right]$,

where $\bar{Q}_{s}^{2}=\frac{C_{F}}{N_{c}} Q_{s}^{2}$ and the anomalous dimension $\gamma\left(Y, \boldsymbol{r}^{2}\right)$ is

$\gamma\left(Y, \boldsymbol{r}^{2}\right)=\frac{1}{2}\left(1+\frac{\xi\left(Y, \boldsymbol{r}^{2}\right)}{\xi\left(Y, \boldsymbol{r}^{2}\right)+\sqrt{2 \xi\left(Y, \boldsymbol{r}^{2}\right)}+7 \zeta(3) c}\right)$,

with $c$ a free parameter (which was fixed in [26] to $c=4$ ) and

$\xi\left(Y, \boldsymbol{r}^{2}\right)=\frac{\ln \left[1 /\left(\boldsymbol{r}^{2} Q_{s 0}^{2}\right)\right]}{(\lambda / 2)\left(Y-Y_{0}\right)}$.

The authors assume that the saturation scale can be expressed by $Q_{s}^{2}(Y)=\Lambda^{2} A^{1 / 3}(1 / x)^{\lambda}$. The form of the anomalous dimension is inspired by the analytical solutions to the BFKL equation [28]. Namely, in the limit $\boldsymbol{r} \rightarrow 0$ with $Y$ fixed we recover the anomalous dimension in the double logarithmic approximation $\gamma \approx 1-\sqrt{1 /(2 \xi)}$. In another limit of large $Y$ with $\boldsymbol{r}$ fixed, Eq. (5) reduces to the expression of the anomalous dimension near the saddle point in the leading logarithmic approximation $\gamma \approx \frac{1}{2}+\frac{\xi}{14 c \zeta(3)}$. Therefore, Eq. (5) mimicks the onset of the geometric scaling region [17,29]. In the calculations of Ref. [26] it is assumed that a characteristic value of $\boldsymbol{r}$ is $\boldsymbol{r} \approx$ $1 /\left(2 k_{T}\right)$ where $k_{T}$ is the transverse momentum of the valence quark and $\gamma$ was approximated by $\gamma\left(Y, \boldsymbol{r}^{2}\right) \approx \gamma\left(Y, 1 /\left(4 k_{T}^{2}\right)\right)$. In the above expressions the parameters $\Lambda=0.6 \mathrm{GeV}$ and 
$\lambda=0.3$ are fixed by DIS data [14]. Moreover, the authors assume $Y_{0}=0.6$. The initial saturation scale used in (6) is defined by $Q_{s 0}^{2}=Q_{s}^{2}\left(Y_{0}\right)$ with $Y_{0}$ being the lowest value of rapidity at which the low- $x$ quantum evolution effects are essential. As demonstrated in Ref. [26] this parameterization is able to describe the $d A u$ RHIC data when the forward dipole cross section is convoluted with the respective fragmentation function and the parton distributions for the deuteron. On the other hand, in Ref. [12] another phenomenological saturation model has been proposed in order to describe the $d A u$ RHIC data (hereafter denoted DHJ model). The basic modification with respect to the KKT model is the parameterization for the anomalous dimension which is now given by

$\gamma\left(Y, \boldsymbol{r}^{2}\right)=\gamma_{s}+\Delta \gamma\left(Y, \boldsymbol{r}^{2}\right)$

where

$$
\Delta \gamma\left(Y, \boldsymbol{r}^{2}\right)=\left(1-\gamma_{s}\right) \frac{\left|\log \frac{1}{\boldsymbol{r}^{2} Q_{T}^{2}}\right|}{\lambda Y+\left|\log \frac{1}{\boldsymbol{r}^{2} Q_{T}^{2}}\right|+d \sqrt{Y}},
$$

with $Q_{T}=Q_{s}(Y)$ a typical hard scale in the process, $\lambda=0.3$ and $d=1.2$. Moreover, $\gamma_{s}=0.63$ is the anomalous dimension for BFKL evolution with saturation boundary condition. Similarly to the KKT model this model is able to describe the $d A u$ RHIC data.

As already discussed in Ref. [27], based on the universality of the hadronic wave function predicted by the CGC formalism, we might expect that the KKT and DHJ parameterizations would also describe the HERA data on proton structure functions in the kinematical region where the saturation effects should be present (small $x$ and low $Q^{2}$ ). However, this expectation fails when the KKT model is applied, as verified in [27]. Here we extend the analysis to the DHJ model without any modification of the parameters fitted at RHIC, only assuming $A=1$ and adjusting the non-perturbative parameter $\sigma_{0}$, which defines the normalization, in order to describe the $F_{2}$ experimental data at $Q^{2}=10 \mathrm{GeV}^{2}$. In Figs. 1 and 2 we present the predictions of the DHJ model for the proton structure function and compare with the ZEUS data [30]. We can see that this parameterization fails for both small and large values of $Q^{2}$. Consequently, the current parameterizations of the forward dipole cross section which are constrained at RHIC are not able to describe the HERA data. An open question is if minimal modifications in these parameterizations allow to describe both sets of data. Following Ref. [31] we consider a modification of the KKT model assuming that the saturation momentum scale is given as in the GBW model, $Y_{0}=4.6, c=0.2$ and that the typical scale in the computation of $\xi\left(Y, r^{2}\right)$ is the photon virtuality. Its predictions (KKTm lines) are presented in Figs. 1 and 2 . It is observed that these modifications imply a quite good description of the HERA data. Similarly, as the $Q^{2}$ evolution of the $F_{2}$ data is not well described by the DHJ model it is possible to improve this model by the modification of the anomalous dimension. Here we propose to modify the DHJ model assuming now that $Q_{T}=Q_{0}=1.0 \mathrm{GeV}$, i.e. that the typical scale is energy independent. It is important to emphasize that this modification preserves the main properties of the anomalous dimension proposed in [12]. Basically, we still have that the anomalous dimension increases logarithmically with $p_{T}$ from $\gamma=\gamma_{s}$ to its asymptotic value $\gamma \approx 1$, while decreasing with $Y$ as $\Delta \gamma \approx 1 / Y$ at very large rapidity. As shown in Figs. 1 and 2 , with this modification our predictions (GKMN lines) agree with the experimental data.

The question which follows is whether the RHIC data are still well reproduced after these modifications. Following Ref. [12] we have calculated the single inclusive hadron production cross section in $d A u$ collisions at different rapidities. We have used the CTEQ5L quark and gluon distributions [32] and the LO KKP quark-hadron fragmentation functions [33]. Our results are presented in Fig. 3 and compared with the BRAHMS data [24]. The KKTm and GKMN predictions are represented by long-dashed and solid curves respectively. As in Ref. [12] we need a $K$-factor in our calculations, since it has been performed at leading order in $\alpha_{s}$. Although the normal-
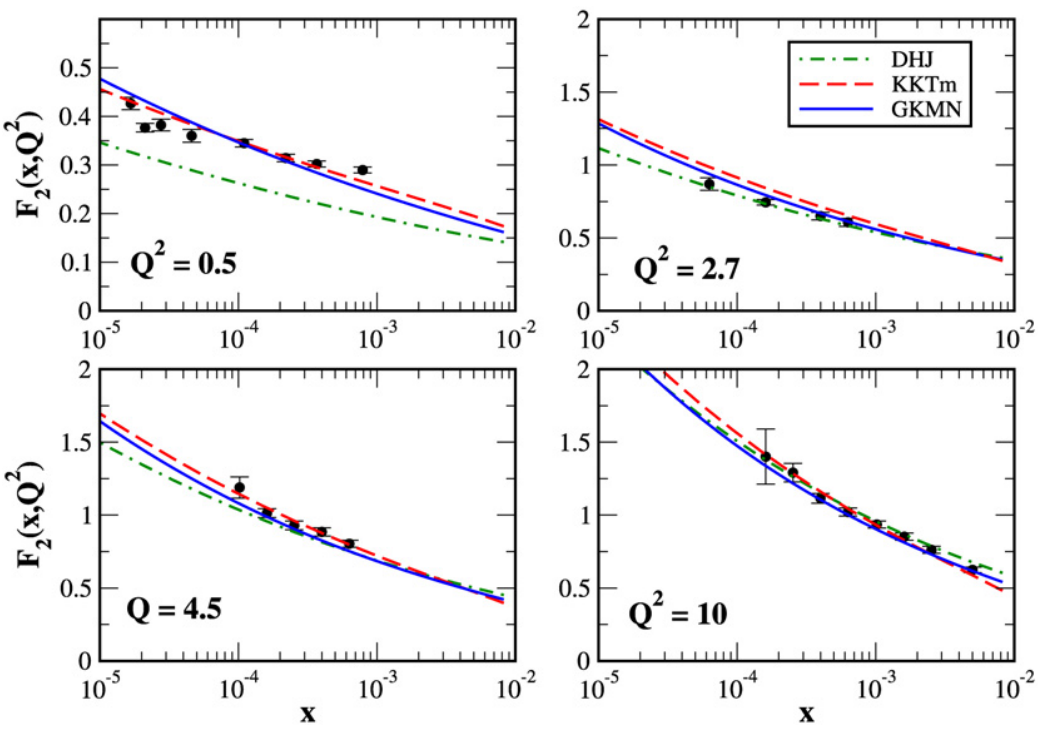

Fig. 1. The proton structure function at different values of the photon virtualities. Data from ZEUS. 


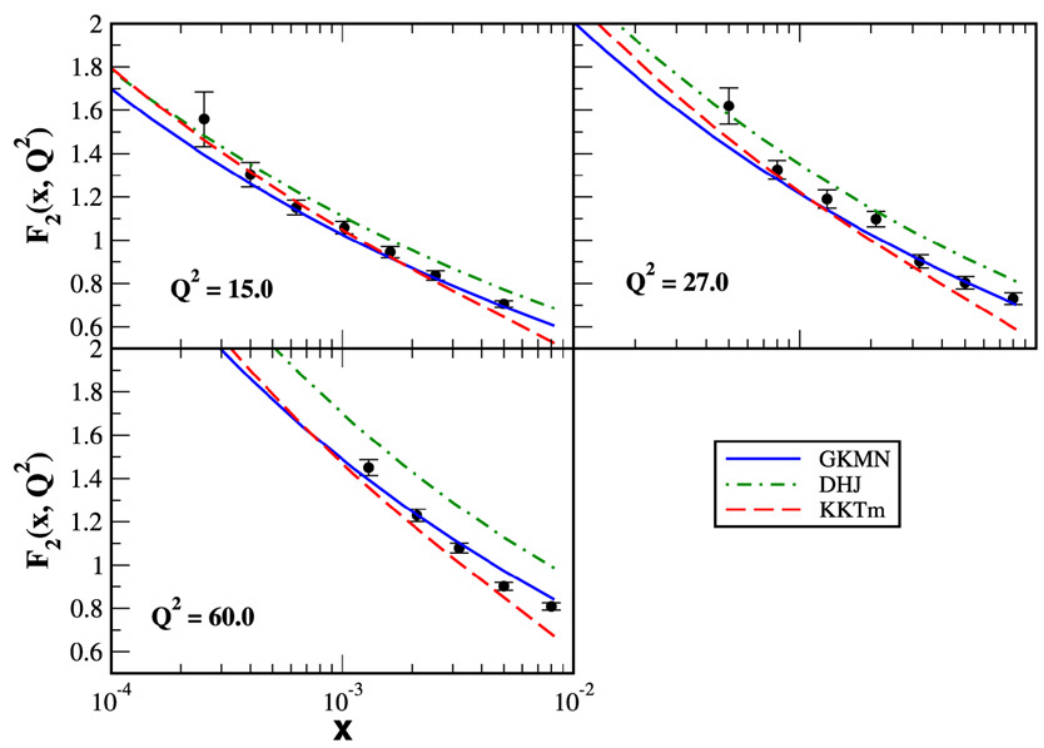

Fig. 2. The proton structure function at different values of the photon virtualities. Data from ZEUS.
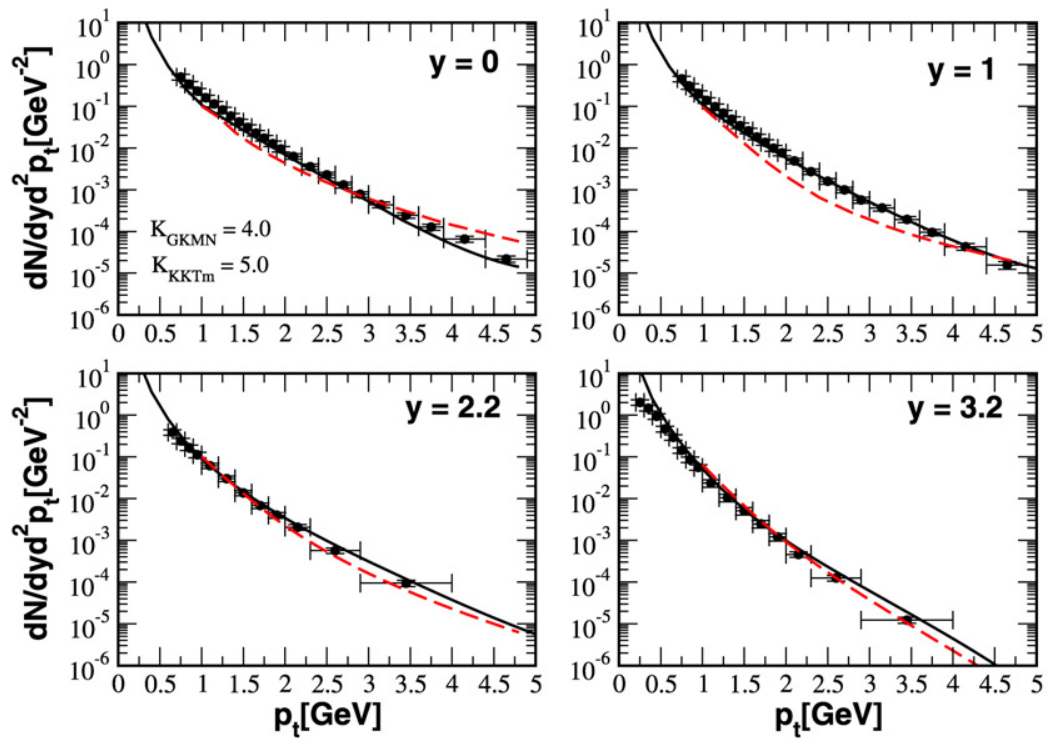

Fig. 3. Comparison of theory and BRAHMS data for minimum-bias $d A u$ collisions at RHIC energy.

ization should be modified by these corrections we expect that the shape of the momentum distributions should not change. Our values of $K$ have been determined so as to reproduce the data at $p_{T}=1.0 \mathrm{GeV}$ and they depend on the parameterization adopted. For KKTm we find a larger value of $K$ than for GKMN. Moreover, while the KKTm parameterization fails to describe the full set of data, the GKMN one is able to reproduce the data quite well even at very small values of $p_{T}$. Consequently, the GKMN model is able to describe the ep HERA and $d A u$ RHIC data in terms of an unique parameterization for the dipole scattering amplitude, which is based on the saturation physics.

Before presenting a summary of our main results, let us briefly discuss the basic properties of the resulting GKMN model (a more detailed analysis will be presented elsewhere). In Fig. 4(a) we present the forward dipole cross section as a function of the scaling variable $r Q_{s}$ for distinct parameterizations. As it can be seen the DHJ, KKTm and GKMN models have a similar behavior. The difference among the models can be demonstrated studying the $Q^{2}$ behavior of the effective anomalous dimension, defined by $\gamma_{\mathrm{eff}}=\frac{d \ln \mathcal{N}\left(r Q_{s}, Y\right)}{d \ln \left(r^{2} Q_{s}^{2} / 4\right)}$ (see similar analyzes in Ref. [31]). In Fig. 4(b) is shown $\gamma_{\text {eff }}$ as a function of the virtuality $Q^{2}$, using the average dipole size as $r=2 / Q$. While the GBW model presents a fast convergence to the DGLAP anomalous dimension at large $Q^{2}$, the IIM parameterization has a mild growth with virtuality, converging to $\gamma \approx 0.85$ at large $Q^{2}$. The KKTm and IIM parameterizations are similar at large $Q^{2}$, but differ at small virtualities, with the KKTm one predicting a smaller value. On the other hand, the predictions of the DHJ and GKMN parameterizations are similar at small $Q^{2}$ and differ at large virtualities. In particular, we have a strict difference between these models in the intermedi- 


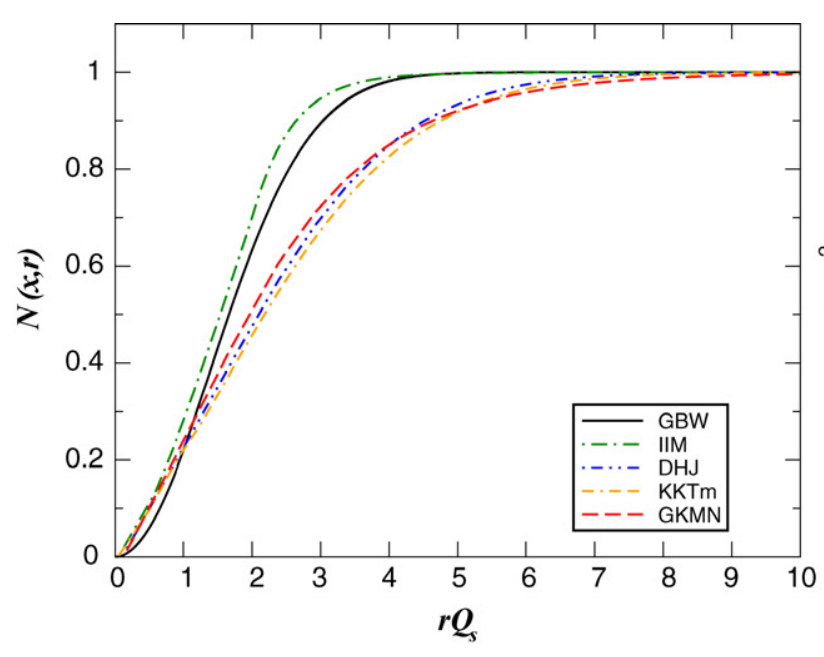

(a)

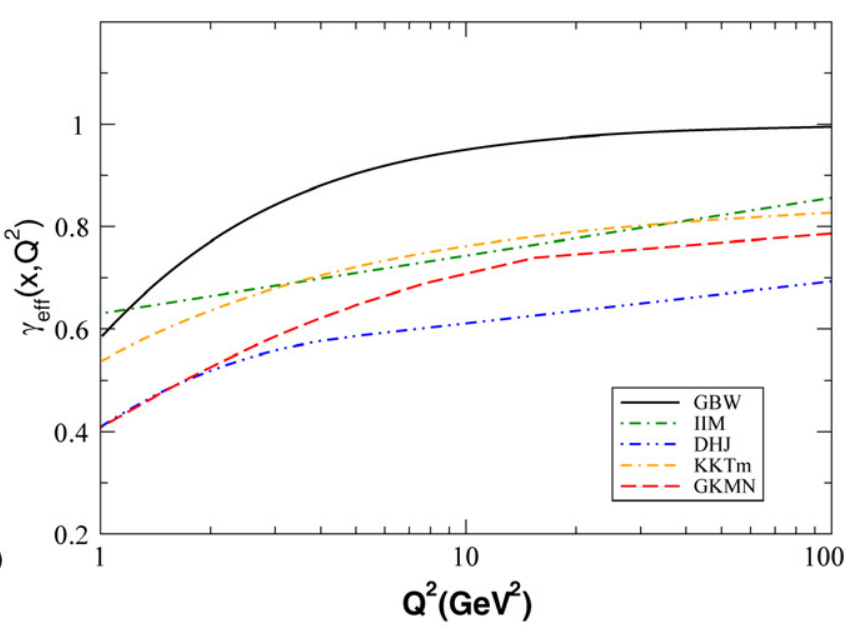

(b)

Fig. 4. (a) Forward dipole cross section as a function of the scaling variable $r Q_{s}$. (b) The $Q^{2}$ behavior of the effective anomalous dimension at $x=3 \times 10^{-4}$.

ate range of virtualities, which can explain why the DHJ model does not describe the $Q^{2}$ evolution of the $F_{2}$ structure function.

As a summary, in this letter we have analyzed current parameterizations for the dipole scattering amplitude which are able to describe separately the $e p$ HERA and $d A u$ RHIC data. We have shown that an unified description using these parameterizations is not possible. We have proposed a modification in the DHJ parameterization for the dipole scattering amplitude, based on saturation physics, which allows to describe simultaneously the $e p$ HERA and $d A u$ RHIC data. This result has been obtained adjusting the normalizations of the dipole cross section and single inclusive hadron cross section and assuming an energy independent typical scale, keeping all other original parameters. A global least $\chi^{2}$ fit of data would change slightly the values of our parameters. This would be a fine tunning which is beyond the scope of this work. We rather prefer to keep the level of fitting accuracy of [12] and emphasize the strategy to reconcile two different sets of data. Apart from this fine tunning, a more detailed theoretical study of the proposed anomalous dimension is necessary. We postpone these improvements for a future publication. Finally, our results demonstrate that an unified description of the experimental data which probes the high energy regime of QCD is possible. This is an important signature of the universality of the saturation physics.

\section{Acknowledgements}

V.P.G. would like to thanks M.A. Betemps for informative and helpful discussions. This work was partially financed by the Brazilian funding agencies CNPq, FAPESP and FAPERGS.

\section{References}

[1] E. Iancu, R. Venugopalan, hep-ph/0303204.

[2] H. Weigert, Prog. Part. Nucl. Phys. 55 (2005) 461.

[3] J. Jalilian-Marian, Y.V. Kovchegov, Prog. Part. Nucl. Phys. 56 (2006) 104.

[4] L. McLerran, R. Venugopalan, Phys. Rev. D 49 (1994) 2233;

L. McLerran, R. Venugopalan, Phys. Rev. D 49 (1994) 3352;

L. McLerran, R. Venugopalan, Phys. Rev. D 50 (1994) 2225.
[5] I.I. Balitsky, Nucl. Phys. B 463 (1996) 99;

I.I. Balitsky, Phys. Rev. Lett. 81 (1998) 2024;

I.I. Balitsky, Phys. Rev. D 60 (1999) 014020;

I.I. Balitsky, Phys. Lett. B 518 (2001) 235;

I.I. Balitsky, A.V. Belitsky, Nucl. Phys. B 629 (2002) 290.

[6] E. Iancu, A. Leonidov, L. McLerran, Nucl. Phys. A 692 (2001) 583; E. Ferreiro, E. Iancu, A. Leonidov, L. McLerran, Nucl. Phys. A 701 (2002) 489.

[7] J. Jalilian-Marian, A. Kovner, L. McLerran, H. Weigert, Phys. Rev. D 55 (1997) 5414;

J. Jalilian-Marian, A. Kovner, H. Weigert, Phys. Rev. D 59 (1999) 014014; J. Jalilian-Marian, A. Kovner, H. Weigert, Phys. Rev. D 59 (1999) 014015; J. Jalilian-Marian, A. Kovner, H. Weigert, Phys. Rev. D 59 (1999) 034007; A. Kovner, J. Guilherme Milhano, H. Weigert, Phys. Rev. D 62 (2000) 114005; H. Weigert, Nucl. Phys. A 703 (2002) 823.

[8] Y.V. Kovchegov, Phys. Rev. D 60 (1999) 034008; Y.V. Kovchegov, Phys. Rev. D 61 (2000) 074018.

[9] M.A. Kimber, J. Kwiecinski, A.D. Martin, Phys. Lett. B 508 (2001) 58; E. Levin, M. Lublinsky, Nucl. Phys. A 696 (2001) 833; M. Lublinsky, Eur. Phys. J. C 21 (2001) 513;

M. Lublinsky, E. Gotsman, E. Levin, U. Maor, Nucl. Phys. A 696 (2001) 851 ;

K. Golec-Biernat, L. Motyka, A.M. Stasto, Phys. Rev. D 65 (2002) 074037;

K. Golec-Biernat, A.M. Stasto, Nucl. Phys. B 668 (2003) 345;

K. Rummukainen, H. Weigert, Nucl. Phys. A 739 (2004) 183;

E. Gotsman, M. Kozlov, E. Levin, U. Maor, E. Naftali, Nucl. Phys. A 742 (2004) 55;

J.L. Albacete, N. Armesto, J.G. Milhano, C.A. Salgado, U.A. Wiedemann, Phys. Rev. D 71 (2005) 014003;

K. Kutak, A.M. Stasto, Eur. Phys. J. C 41 (2005) 343;

G. Chachamis, M. Lublinsky, A. Sabio Vera, Nucl. Phys. A 748 (2005) 649;

T. Ikeda, L. McLerran, Nucl. Phys. A 756 (2005) 385;

C. Marquet, G. Soyez, Nucl. Phys. A 760 (2005) 208;

R. Enberg, K. Golec-Biernat, S. Munier, Phys. Rev. D 72 (2005) 074021.

[10] N.N. Nikolaev, B.G. Zakharov, Z. Phys. C 49 (1991) 607; N.N. Nikolaev, B.G. Zakharov, Z. Phys. C 53 (1992) 331.

[11] V. Barone, E. Predazzi, High-Energy Particle Diffraction, Springer-Verlag, Berlin, 2002.

[12] A. Dumitru, A. Hayashigaki, J. Jalilian-Marian, Nucl. Phys. A 765 (2006) 464;

A. Dumitru, A. Hayashigaki, J. Jalilian-Marian, Nucl. Phys. A 770 (2006) 57. 
[13] V.N. Gribov, L.N. Lipatov, Sov. J. Nucl. Phys. 15 (1972) 438;

G. Altarelli, G. Parisi, Nucl. Phys. B 126 (1977) 298;

Yu.L. Dokshitzer, Sov. Phys. JETP 46 (1977) 641.

[14] K. Golec-Biernat, M. Wüsthoff, Phys. Rev. D 59 (1999) 014017; K. Golec-Biernat, M. Wüsthoff, Phys. Rev. D 60 (1999) 114023.

[15] J. Bartels, K. Golec-Biernat, H. Kowalski, Phys. Rev. D 66 (2002) 014001.

[16] H. Kowalski, D. Teaney, Phys. Rev. D 68 (2003) 114005.

[17] E. Iancu, K. Itakura, S. Munier, Phys. Lett. B 590 (2004) 199.

[18] H. Kowalski, L. Motyka, G. Watt, hep-ph/0606272.

[19] K. Golec-Biernat, S. Sapeta, Phys. Rev. D 74 (2006) 054032.

[20] A.M. Staśto, K. Golec-Biernat, J. Kwieciński, Phys. Rev. Lett. 86 (2001) 596.

[21] C. Marquet, L. Schoeffel, Phys. Lett. B 639 (2006) 471.

[22] V.P. Goncalves, M.V.T. Machado, Phys. Rev. Lett. 91 (2003) 202002.

[23] V.P. Goncalves, M.V.T. Machado, hep-ph/0607125.

[24] I. Arsene, et al., BRAHMS Collaboration, Phys. Rev. Lett. 91 (2003) 072305;

I. Arsene, et al., BRAHMS Collaboration, Phys. Rev. Lett. 93 (2004) 242303;

I. Arsene, et al., BRAHMS Collaboration, Phys. Rev. Lett. 94 (2005) 032301;
I. Arsene, et al., BRAHMS Collaboration, nucl-ex/0410020.

[25] R. Baier, A. Kovner, U.A. Wiedemann, Phys. Rev. D 68 (2003) 054009;

J. Jalilian-Marian, Y. Nara, R. Venugopalan, Phys. Lett. B 577 (2003) 54;

D. Kharzeev, Y.V. Kovchegov, K. Tuchin, Phys. Rev. D 68 (2003) 094013;

J.L. Albacete, N. Armesto, A. Kovner, C.A. Salgado, U.A. Wiedemann, Phys. Rev. Lett. 92 (2004) 082001;

E. Iancu, K. Itakura, D.N. Triantafyllopoulos, Nucl. Phys. A 742 (2004) 182.

[26] D. Kharzeev, Y.V. Kovchegov, K. Tuchin, Phys. Lett. B 599 (2004) 23.

[27] M.S. Kugeratski, V.P. Goncalves, F.S. Navarra, Eur. Phys. J. C 44 (2005) 577.

[28] L.N. Lipatov, Sov. J. Nucl. Phys. 23 (1976) 338;

E.A. Kuraev, L.N. Lipatov, V.S. Fadin, JETP 45 (1977) 1999;

I.I. Balitskii, L.N. Lipatov, Sov. J. Nucl. Phys. 28 (1978) 822.

[29] E. Iancu, K. Itakura, L. McLerran, Nucl. Phys. A 708 (2002) 327.

[30] S. Chekanov, et al., ZEUS Collaboration, Eur. Phys. J. C 21 (2001) 443.

[31] M.V.T. Machado, Eur. Phys. J. C 47 (2006) 365.

[32] H.L. Lai, et al., CTEQ Collaboration, Eur. Phys. J. C 12 (2000) 375.

[33] B.A. Kniehl, G. Kramer, B. Potter, Nucl. Phys. B 582 (2000) 514. 